\title{
An Nd:YAG dual-channel laser system with Q - modulation for direct interference lithography
}

\author{
J. Marczak, A. Rycyk, A. Sarzyński, M. Strzelec, ${ }^{*}$ K. Czyż \\ Institute of Optoelectronics, Military University of Technology, Gen. S. Kaliskiego 2, 00-908 Warszawa
}

Received October 03, 2013; accepted March 30, 2014; published March 31, 2014

\begin{abstract}
A dual-channel laser system consisting of an Nd:YAG generator with Q - modulation and a "p - branch" type unstable resonator has been presented in the article. The laser assembly has been configured in such a way as to constitute the arms of a Mach-Zehnder interferometer. The regulation of the amplification coefficient in both amplifying channels allows for quick and easy changes of the output energy in each channel up to a value of approximately $1.5 \mathrm{~J}$, with a laser pulse duration of 8-10ns. The Mach-Zehnder configuration of the interference assembly allowed for quick and easy changes of the angle between the interfering laser beams.
\end{abstract}

The use of lasers for scientific research and industrial application is associated directly with the unique properties of laser radiation. High temporal and spatial coherence achieved in lasers allows for extreme orientation of the laser beam and its focussing. The monochromaticity of laser light along with the wavelength tuning ability and conversion into higher harmonics allows for highly selective excitation of numerous materials. In practice, all these properties of laser light are also used for topographic and microstructural changes, meaning the modification of the surface layers according to a specified pattern [1]. The possibility of efficient and economical creation of well defined regular surface structures may be a successful way to significantly improve the existing or create new properties of numerous materials [2].

In the last decade the direct interference lithography method utilising lasers of high power density between $\mathrm{MW} / \mathrm{cm}^{2}$ and $\mathrm{GW} / \mathrm{cm}^{2}$ has been used for periodic surface modifications of virtually any solid materials [3-6]. This method allows in a direct manner for the creation of surface structures in the micro- and sub- micron scale with a well defined long-range arrangement.

The impact of such strong pulse laser radiation on the surface of metals, semiconductors, isolators, ceramics and polymers results in direct shaping of phases and defects in the micro- and sub- micron scale, with the occurrence of well known metallurgical phenomena, such as: heating coupled with phase transitions, stress relief, melting, recrystallisation or ablation of the material.

\footnotetext{
*E-mail: mstrzelec@wat.edu.pl
}
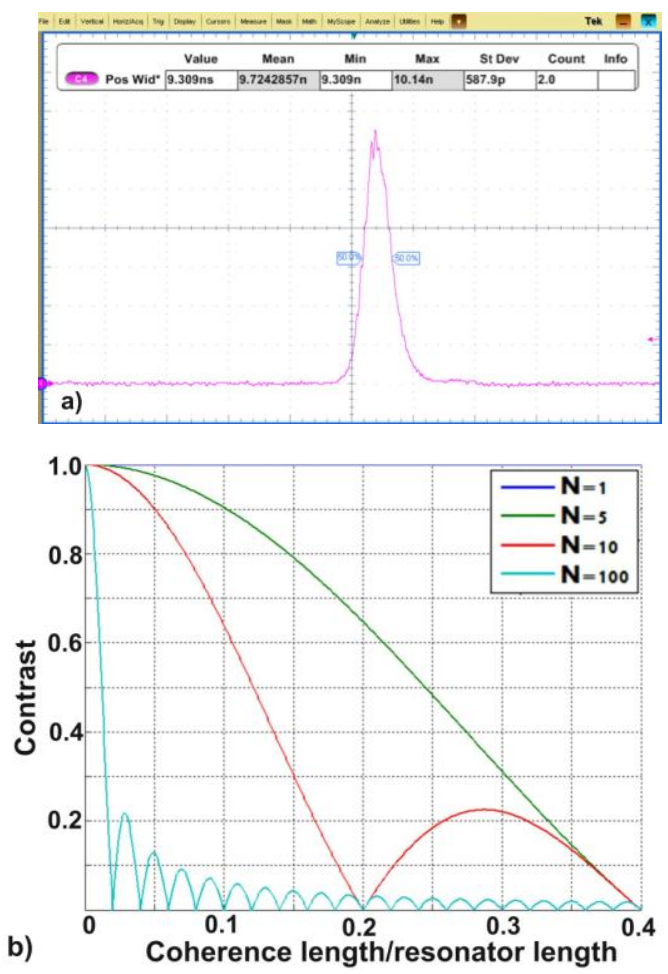

Fig. 1. a) A laser pulse time duration oscillogram. b) Interference contrast of a laser emitting 1, 5, 10 and 100 longitudinal modes.

A dual-channel laser system consisting of a Nd:YAG generator with Q - modulation and a "p - branch" type unstable resonator has been constructed for direct laser interference lithography. The output of the laser beam from a generator has been realised via a quarter-wave plate and a dielectric polariser, and a diaphragm placed inside the resonator ensured a primary transverse mode. The output energy ranged between approximately 50 and $60 \mathrm{~mJ}$, and the pulse duration depending on the amount of pumping energy ranged between 8 and 10ns (Fig. 1a). In the practical laser arrangements utilising an yttriumaluminium crystal supplemented by neodymium ions, the temporal coherence of the generated beams is sufficient even during the generation of several or about a dozen longitudinal modes, which has been shown in Fig. $1 \mathrm{~b}$. 


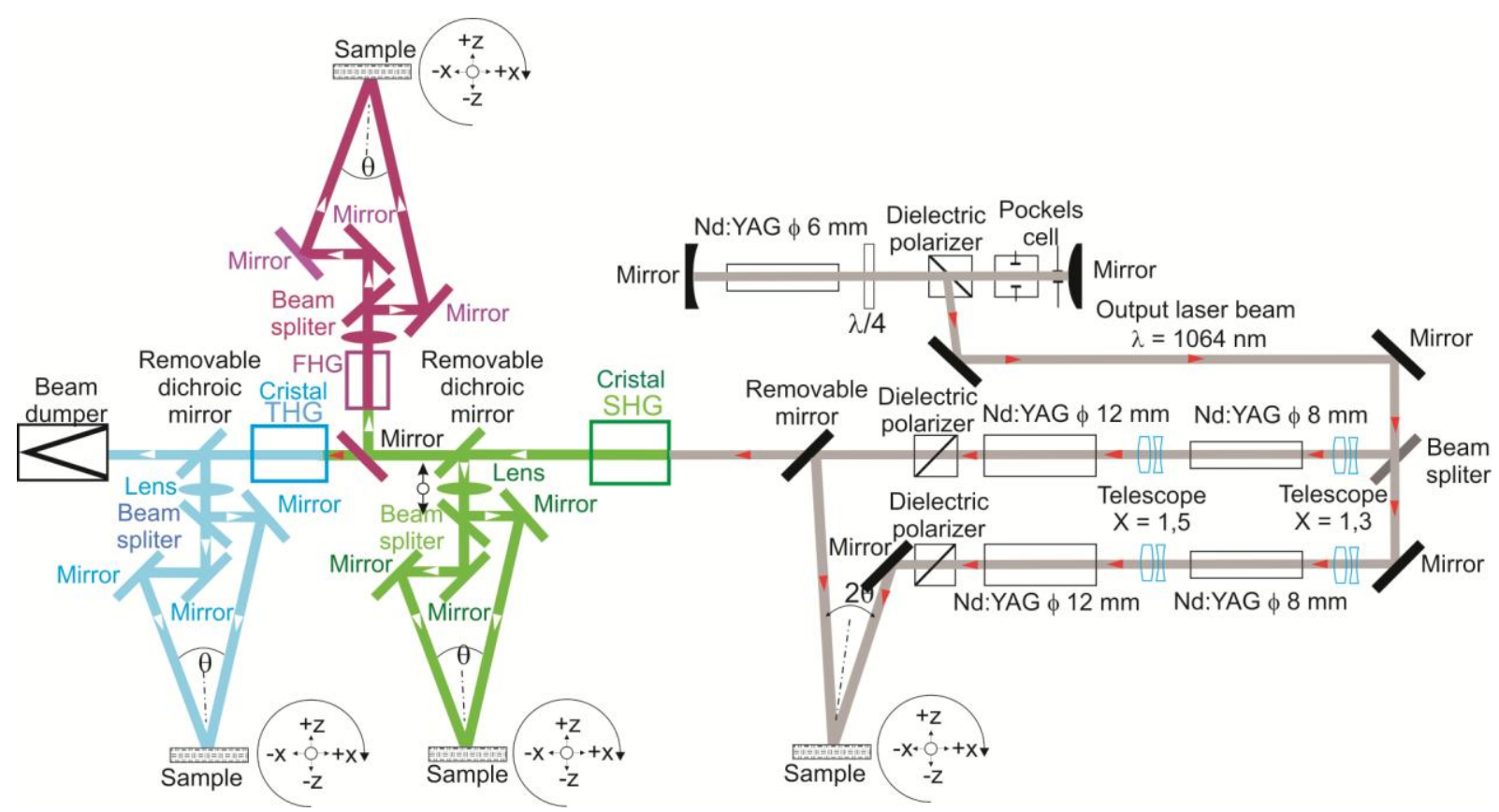

Fig. 2. General scheme of a dual-channel laser arrangement (on the right-hand side) and optical schemes of the combination of two laser beams after conversion into the second (SHG), third (THG) and fourth (FHG) harmonic (on the left-hand side).

After the release of the laser pulse from the generator, amplitude splitting into two energetically equal laser beams by means of a dielectric mirror took place. Both laser beams were introduced into two amplifiers in each channel. The laser rod diameters inside the amplifiers equalled $8 \mathrm{~mm}$ and $12 \mathrm{~mm}$, respectively. Polarisers were used additionally at the output of each amplifying channel in order to eliminate the parasitic polarisation component created as a result of thermal birefringence in laser rods excited by flash lamps. The scheme of a dual-channel laser system, constituting an "active" Mach-Zehnder interferometer has been presented in Fig. 2.

The quick and easy changes of the angle between the interfering laser beams constitute the primary advantage of such a configuration of laser assembly an "active" Mach-Zehnder interferometer. This allows for the creation of periodic structures on any given surface, even of refractory materials, by the proper change of the amplification coefficient in the amplifying channels, and the area of impact is close to the output diameter of the final amplifier. Such a change in the magnitude of energy density in the beam covering area while maintaining a constant period of the linear grid has been shown in Fig. 3. Exemplary three-dimensional images of the resulting linear structures have been shown in Figs. 4-5.

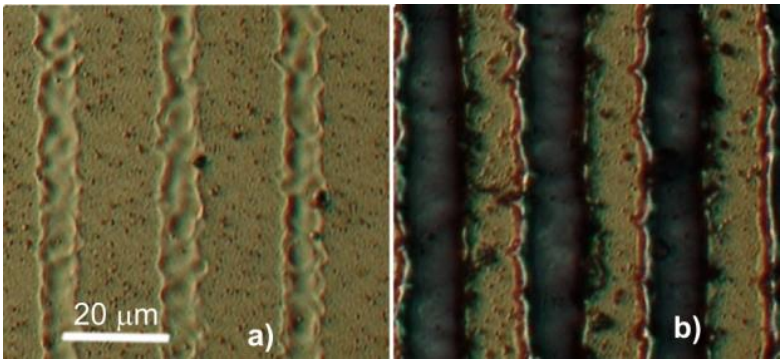

Fig. 3. A picture presenting the constant period of diffraction grid maintained during the change in width and depth of resulting depressions, created in a 50nm layer of Au deposited on an approximately $50 \mathrm{~nm}$ thick layer of Cr: a) fluency $300 \mathrm{~mJ} / \mathrm{cm}^{2} ;$ b) fluency $550 \mathrm{~mJ} / \mathrm{cm}^{2}$.

The lower, theoretical limit of the distance between interference fringes equals half the wavelength of the illuminating radiation. In practice, the total angle between the interfering laser beams does not exceed $120^{\circ}$, and the minimal distance between the fringes is higher than $\lambda / 2$, which is a result of the impact of the parameters of the laser beam itself (the degree of coherence and polarisation) as well as the physical and chemical properties of the processed material. The depth of the resulting structures depends on the energy density and the laser pulse duration. Adding the converters of the $\mathrm{Nd}$ :YAG laser radiation frequency into higher harmonics makes it possible to adjust the laser radiation wavelength to the attenuation coefficient of the given material. 


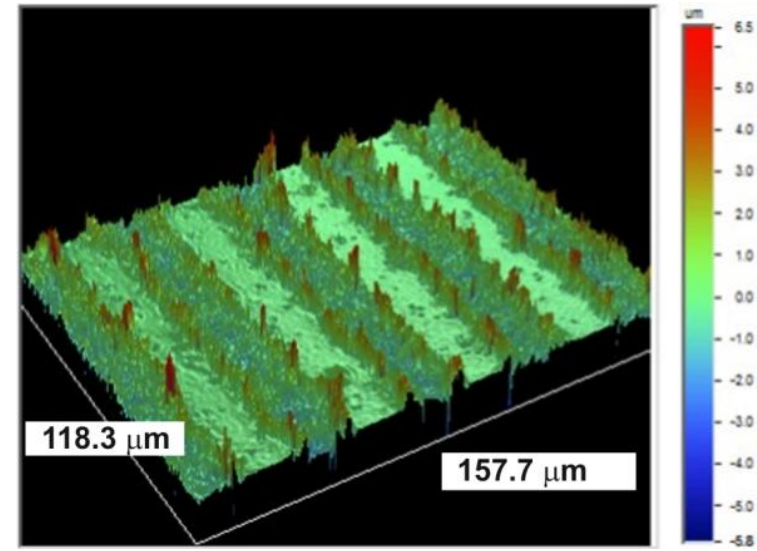

Fig. 4. A spatial image of the 1D interference structures on aluminium from a Vecco profilometer. The output energy from one channel is approximately $950 \mathrm{~mJ}$, the surface area diameter $-12 \mathrm{~mm}$.

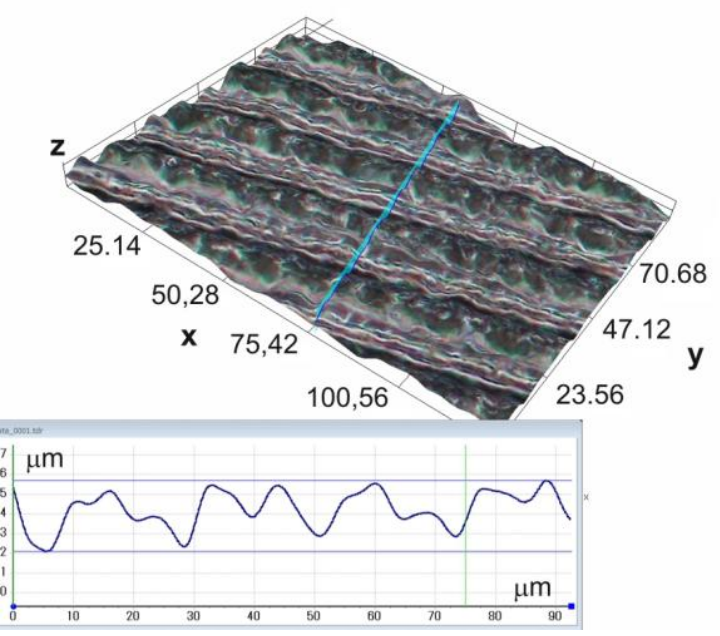

Fig. 5. A spatial image and profile of an interference structure obtained for a three-layered $\mathrm{Cr}-\mathrm{Cu}-\mathrm{Al}$ sample, with the thicknesses of respectively: $50 \mathrm{~nm}, 300 \mathrm{~nm}$ and $100 \mathrm{~nm}$ lying on a $1 \mathrm{~mm}$ thick quartz plate. Fluency approximately $600 \mathrm{~mJ} / \mathrm{cm}^{2}$.

Photonic methods of creating micro- and submicronic structures have a number of advantages due to remote and contactless processing, flexibility during material processing and precise energy dosage in order to obtain one-, two- and three-dimensional periodic structures. Such structures on the surface of metals, semiconductors, dielectrics or polymers allow for generation of new properties for materials with very specific electrical, mechanical or chemical characteristics. Depending on the specified material parameters and structure morphology, new systems may be realised, such as bio-sensors [7], microflow systems [8-10], templates for biological applications [11] and photonic structures [12]. The first 1D and 2D profiles created by means of the laser system described in the article on the surface of thin DLC layers on a silicone substrate are currently researched regarding their use in scaffolds for the growth of biological cells. The initial results indicate that the modification of such biocompatible layers improves and strengthens mutual interaction between the cells and the surface and, consequently, their traction, growth and direction of orientation [13].

The work is funded in the frames of the National Science Centre project no. 2326/B/T02/2011/40 "Laser interference shaping of surface layers in metals".

\section{References}

[1] D. Xia, Z. Ku, S.C. Lee, S.R.J. Brueck, Adv. Mater. 23, 147 (2011).

[2] B. Raeymaekers, E. Izhak, F.E. Talka, Tribol. Lett. 27, 89 (2007).

[3] F. Mucklich, A. Lasagni, C. Daniel, Intermetallics 13, 437 (2005).

[4] R. Major, K. Maksymow, J. Marczak, J.M. Lackner, M. Kot, B. Major, Bull. Pol. Acad. Sci-Tech. 60, 337 (2012).

[5] J. Marczak, A. Rycyk, A. Sarzyński, M. Strzelec, J. Kusiński, R. Major, Proc. SPIE 8703, 87030F (2013).

[6] C. Daniel, J. Drummond, R.A. Giordano, J. Am. Ceram. Soc. 91 3455 (2008).

[7] N. Ganesh, I.D. Block, B.T. Cunningham, Appl. Phys. Lett. 89, 023901 (2006).

[8] H. Yu, O. Balogun, B. Li, T.W. Murray, X. Zhang, J. Micromech. Microeng. 14, 1576 (2004).

[9] W.H. Ryu, S.W. Min, K.E. Hammerick, M. Vyakarnam, R.S. Greco, F.B. Prinz, R.J. Fasching, Biomaterials 28, 1174 (2007)

[10] F.B. Prinz, R.S. Smith, Nanoscale Technology in Biological Systems (Boca Raton, CRC Press 2005).

[11] J. Zhang et al., Biomaterials 27, 5734 (2006).

[12] I.B. Divliansky et al., Appl. Phys. Lett. 79, 3392 (2001).

[13] J. Marczak, Invited Presentation. Laser interference patterning of DLC layers for directed migration and growth of smooth muscle cell depositions. EMRS 2013 Fall Meeting 2013: http://www.emrsstrasbourg.com/index.php?option=com abstract\&task=view\&id=2 28\&day $=2013-09$ -

$\underline{18 \& \text { year }=2013 \& \text { Itemid }=99999999 \& i d \_ \text {season }=10 .}$. 